\title{
MULTIPLICATION OPERATORS AND DYNAMICAL SYSTEMS
}

\section{R. K. SINGH and JASBIR SINGH MANHAS}

(Received 27 June 1990)

Communicated by A. J. Pryde

\begin{abstract}
Let $X$ be a completely regular Hausdorff space, let $V$ be a system of weights on $X$ and let $T$ be a locally convex Hausdorff topological vector space. Then $C V_{b}(X, T)$ is a locally convex space of vector-valued continuous functions with a topology generated by seminorms which are weighted analogues of the supremum norm. In the present paper we characterize multiplication operators on the space $C V_{b}(X, T)$ induced by operator-valued mappings and then obtain a (linear) dynamical system on this weighted function space.
\end{abstract}

1991 Mathematics subject classification (Amer. Math. Soc.): 34 C 35, 46 E 40, 47 B 38.

Keywords and phrases: system of weights, locally convex spaces, multiplication operators, dynamical systems.

\section{Introduction}

Let $X$ be a non-empty set, let $T$ be a topological algebra and let $L(X, T)$ be the linear space of all functions from $X$ to $T$. Let $F(X, T)$ be a topological vector subspace of $L(X, T)$. Let $\psi$ be a mapping on $X$ such that $\psi f \in$ $L(X, T)$ whenever $f \in F(X, T)$. This gives rise to a linear transformation $M_{\psi}: F(X, T) \rightarrow L(X, T)$ defined as $M_{\psi} f=\psi f$, where the product of functions is defined pointwise. In case $M_{\psi}$ takes $F(X, T)$ into itself and is continuous, it is called a multiplication operator on $F(X, T)$ induced by the mapping $\psi$.

This paper is a continuation of our earlier paper [8] in which we have studied multiplication operators on weighted spaces of vector-valued con-

(C) 1992 Australian Mathematical Society $0263-6115 / 92 \$ A 2.00+0.00$ 
tinuous functions induced by scalar-valued and vector-valued mappings. In the present paper we concentrate on the study of multiplication operators on weighted spaces of vector-valued mappings induced by operator-valued mappings and then we endeavor to study a (linear) dynamical system on these function spaces.

\section{Preliminaries}

Let $X$ be a completely regular Hausdorff space, let $T$ be a Hausdorff locally convex topological vector space over $\mathbb{C}$ and let $C(X, T)$ be the vector space of all continuous functions from $X$ into $T$. By $\operatorname{cs}(T)$ we mean the set of all continuous seminorms on $T$, and $B(T)$ denotes the set of all continuous linear operators on $T$. By a system of weights we mean a set $V$ of non-negative upper-semicontinuous functions on $X$ such that, given any $x \in X$, there is some $v \in V$ for which $v(x)>0$ and for every pair $u, v \in V$ and $\alpha>0$, there exists $w \in V$ so that $\alpha u \leq w$ and $\alpha v \leq w$ (point wise on $X$ ).

Now we consider the following vector space of vector-valued continuous functions:

$$
C V_{b}(X, T)=\{f \in C(X, T): v f(X) \text { is bounded in } T \text { for all } v \in V\} .
$$

Now, let $v \in V, q \in \operatorname{cs}(T)$ and $f \in C(X, T)$. If we put $\|f\|_{v, q}=$ $\operatorname{Sup}\{v(x) q(f(x)): x \in X\}$, then $\|\cdot\|_{v, q}$ is a seminorm on $C V_{b}(X, T)$ and the family $\left\{\|\cdot\|_{v, q}: v \in V \quad q \in \operatorname{cs}(T)\right\}$ defines a locally convex topology on $C V_{b}(X, T)$.

In case $T=\mathbb{C}$, we shall omit $T$ from our notation and write $C V_{b}(X)$ in place of $C V_{b}(X, \mathbb{C})$. We also write $\|\cdot\|_{v}$ in place of $\|\cdot\|_{v, q}$ for each $v \in V$, where $q(z)=|z|, z \in \mathbb{C}$. We shall denote by $B_{v, q}$ the closed unit ball corresponding to the seminorm $\|\cdot\|_{v, q}$. In case $T=(T, q)$, any normed linear space, we simply write $B_{v}$. We refer to the papers of Bierstedt $[1,2]$ and Prolla [7] for more details and examples of these function spaces.

Let $\mathscr{F}$ be the family of all bounded subsets of $T$ and let $M \in \mathscr{F}$ and $p \in \operatorname{cs}(T)$. If we define the function

$$
S_{M, p}: B(T) \rightarrow \mathbb{R}^{+} \text {as } S_{M, p}(A)=\operatorname{Sup}\{p(A(y)): y \in M\}
$$

then $S_{M, p}$ is a seminorm on $B(T)$ and the family $\left\{S_{M, p}: M \in \mathscr{F}, p \in\right.$ $\operatorname{cs}(T)\}$ defines a locally convex topology on $B(T)$ which we call the topology of uniform convergence on bounded sets and denote by $\mathscr{U}$. Thus $(B(T), \mathscr{U})$ is a locally convex topological vector space of continuous linear operators on 
$T$. For more details of these topologies on the spaces of linear operators we refer to Grothendieck [4] and Kothe [5].

\section{Functions inducing multiplication operators}

Throughout this section we will work under the following modest requirements, while developing our characterisation of an operator-valued mapping $\psi: X \rightarrow B(T)$ which induces a multiplication operator on $C V_{b}(X, T)$ :

(2.a) $X$ is a completely regular Hausdorff space;

(2.b) $T$ is a Hausdorff locally convex topological vector space;

(2.c) $V$ is a system of weights on $X$.

In the following theorem we characterise operator-valued mappings which induce multiplication operators on $C V_{b}(X, T)$.

2.1. THEOREM. Let $\psi: X \rightarrow B(T)$ be an operator-valued continuous function. Then $M_{\psi}: C V_{b}(X, T) \rightarrow C V_{b}(X, T)$ is a multiplication operator if and only if for every $v \in V$ and $p \in \operatorname{cs}(T)$, there exist $u \in V$ and $q \in \operatorname{cs}(T)$ such that $v(x) p(\psi(x) y) \leq u(x) q(y)$, for every $x \in X$ and $y \in T$.

Proof. First, let us suppose that for every $v \in V$ and $p \in \operatorname{cs}(T)$, there exist $u \in V$ and $q \in \operatorname{cs}(T)$ such that

$$
v(x) p(\psi(x) y) \leq u(x) q(y), \quad \text { for every } x \in X \text { and } y \in T .
$$

Then we shall show that $M_{\psi}$ is a continuous linear operator on $C V_{b}(X, T)$. First of all, we show that $M_{\psi}$ is an into map. Let $\left\{x_{\alpha}: \alpha \in \Delta\right\}$ be a net in $X$ such that $x_{\alpha} \rightarrow x$. To show that $\psi\left(x_{\alpha}\right) f\left(x_{\alpha}\right) \rightarrow \psi(x) f(x)$ in $T$, it suffices to show that for every $p \in \operatorname{cs}(T)$ and $\varepsilon>0$, there exists $\alpha_{0} \in \Delta$ such that

$$
p\left(\psi\left(x_{\alpha}\right) f\left(x_{\alpha}\right)-\psi(x) f(x)\right)<\varepsilon, \quad \text { for every } \alpha \geq \alpha_{0} .
$$

Now,

$$
\begin{aligned}
p\left(\psi\left(x_{\alpha}\right) f\left(x_{\alpha}\right)-\psi(x) f(x)\right) \leq & p\left[\left(\psi\left(x_{\alpha}\right)-\psi(x)\right)\left(f\left(x_{\alpha}\right)\right)\right] \\
& +p\left[\psi(x)\left(f\left(x_{\alpha}\right)-f(x)\right)\right] .
\end{aligned}
$$

Since the set $\left\{f\left(x_{\alpha}\right): \alpha \in \Delta\right\}$ is bounded in $T$, for every $p \in \operatorname{cs}(T)$ and $\varepsilon>0$, there exists $\alpha_{1} \in \Delta$ such that

$$
p\left[\left(\psi\left(x_{\alpha}\right)-\psi(x)\right)\left(f\left(x_{\alpha}\right)\right)\right]<\varepsilon / 2, \quad \text { for every } \alpha \geq \alpha_{1} .
$$

Again, since $\psi(x)$ is a continuous linear operator on $T$, for every $p \in \operatorname{cs}(T)$ and $\varepsilon>0$, there exists a neighbourhood $W$ of the origin in $T$ such that 
$p(\psi(x) y)<\varepsilon / 2$ for every $y \in W$. Since $f$ is continuous, there exists $\alpha_{2} \in \Delta$ such that $f\left(x_{\alpha}\right)-f(x) \in W$, for $\alpha \geq \alpha_{2}$ and consequently

$$
\left.p\left[\psi(x) f\left(x_{\alpha}\right)-f(x)\right)\right]<\varepsilon / 2, \quad \text { for every } \alpha \geq \alpha_{2} .
$$

Let $\alpha_{0} \in \Delta$ be such that $\alpha_{1} \leq \alpha_{0}$ and $\alpha_{2} \leq \alpha_{0}$. Then from (ii) and (iii) it follows that

$$
p\left(\psi\left(x_{\alpha}\right) f\left(x_{\alpha}\right)-\psi(x) f(x)\right) \leq \varepsilon, \quad \text { for every } \alpha \geq \alpha_{0} .
$$

This proves the continuity of $\psi f$. Further, let $v \in V, p \in \operatorname{cs}(T)$ and $f \in C V_{b}(X, T)$. The

$\|\psi f\|_{v, p}=\operatorname{Sup}\{v(x) p(\psi(x) f(x)) x \in X\} \leq \operatorname{Sup}\{u(x) q(f(x)): x \in X\}<\infty$.

This implies that $\psi f \in C V_{b}(X, T)$. Clearly $M_{\psi}$ is linear on $C V_{b}(X, T)$. In order to prove the continuity of $M_{\psi}$ on $C V_{b}(X, T)$, it is enough to show that $M_{\psi}$ is continuous at the origin. For this, suppose $\left\{f_{\alpha}\right\}$ is a net in $C V_{b}(X, T)$ such that $\left\|f_{\alpha}\right\|_{v, p} \rightarrow 0$, for every $v \in V$ and $p \in \operatorname{cs}(T)$.

$$
\begin{aligned}
\left\|M_{\psi} f_{\alpha}\right\|_{v, p} & =\operatorname{Sup}\left\{v(x) p\left(\psi(x) f_{\alpha}(x)\right): x \in X\right\} \leq \operatorname{Sup}\left\{u(x) q\left(f_{\alpha}(x)\right): x \in X\right\} \\
& =\left\|f_{\alpha}\right\|_{u, q} \rightarrow 0 .
\end{aligned}
$$

This proves the continuity of $M_{\psi}$ at the origin and hence $M_{\psi}$ is continuous on $C V_{b}(X, T)$.

Conversely, suppose $M_{\psi}$ is a continuous linear operator on $C V_{b}(X, T)$. We shall show that for every $v \in V$ and $p \in \operatorname{cs}(T)$, there exist $u \in V$ and $q \in \operatorname{cs}(T)$ such that

$$
v(x) p(\psi(x) y) \leq u(x) q(y), \quad \text { for every } x \in X \text { and } y \in T .
$$

Let $v \in V$ and $p \in \operatorname{cs}(T)$. Since $M_{\psi}$ is continuous at the origin, there exist $u \in V$ and $q \in \operatorname{cs}(T)$ such that $M_{\psi}\left(B_{u, q}\right) \subseteq B_{v, p}$. We claim that

$$
v(x) p(\psi(x) y) \leq 2 u(x) q(y), \quad \text { for every } x \in X \text { and } y \in T \text {. }
$$

Take $x_{0} \in X, y_{0} \in T$ and set $u\left(x_{0}\right) q\left(y_{0}\right)=\varepsilon$. In case $\varepsilon>0$, the set $G=\left\{x \in X: u(x) q\left(y_{0}\right)<2 \varepsilon\right\}$ is an open neighbourhood of $x_{0}$. Thus, according to [6, Lemma 2], there exists $f \in C V_{b}(X)$ such that $0 \leq f \leq 1$, $f\left(x_{0}\right)=1$ and $f(X-G)=0$. Define $g(x)=f(x) y_{0}$, for every $x \in X$. Then clearly $g \in C V_{b}(X, T)$ and for every $p \in \operatorname{cs}(T), 0 \leq(p \circ g) \leq p\left(y_{0}\right)$, $(p \circ g)\left(x_{0}\right)=p\left(y_{0}\right)$ and $(p \circ g)(X-G)=0$. Let $h=\left(2 u\left(x_{0}\right) q\left(y_{0}\right)\right)^{-1} g$. Then clearly $h \in B_{u, q}$ and this yields that $\psi h \in B_{v, p}$. Hence $v(x) p(\psi(x) h(x) \leq$ 1 , for every $x \in X$. From this, it follows that

$$
v(x) p(\psi(x) g(x)) \leq 2 u\left(x_{0}\right) q\left(y_{0}\right), \quad \text { for every } x \in X .
$$

This implies that

$$
v\left(x_{0}\right) p\left(\psi\left(x_{0}\right) y_{0}\right) \leq 2 u\left(x_{0}\right) q\left(y_{0}\right)
$$


On the other hand, suppose $u\left(x_{0}\right) q\left(y_{0}\right)=0$. Then the following three cases arise:

(i) $u\left(x_{0}\right)=0, \quad q\left(y_{0}\right) \neq 0$;

(ii) $u\left(x_{0}\right) \neq 0, \quad q\left(y_{0}\right)=0$;

(iii) $u\left(x_{0}\right)=0, \quad q\left(y_{0}\right)=0$.

Let us suppose that (i) holds and let $v\left(x_{0}\right) p\left(\psi\left(x_{0}\right) y_{0}\right)>0$. Put $\varepsilon=$ $v\left(j x_{0}\right) p\left(\psi\left(x_{0}\right) y_{0}\right) / 2$. Then $G=\left\{x \in X: u(x) q\left(y_{0}\right)<\varepsilon\right\}$ is an open neighbourhood of $x_{0}$ and hence again by [6, Lemma 2], there exists $f \in C V_{b}(X)$ such that $0 \leq f \leq 1, f\left(x_{0}\right)=1$ and $f(X-G)=0$. Again, define $g(x)=f(x) y_{0}$, for every $x \in X$. Then clearly $g \in C V_{b}(X, T)$ and for every $p \in \operatorname{cs}(T), 0 \leq(p \circ g) \leq p\left(y_{0}\right),(p \circ g)\left(x_{0}\right)=p\left(y_{0}\right)$ and $(p \circ g)(X-G)=0$. Consider $h=\varepsilon^{-1} g$. Then $h \in B_{u, q}$ and therefore $\psi h \in B_{v, p}$. Hence $v(x) p(\psi(x) h(x)) \leq 1$ for every $x \in X$. This implies that

$$
v(x) p(\psi(x) g(x)) \leq \frac{v\left(x_{0}\right) p\left(\psi\left(x_{0}\right) y_{0}\right)}{2}, \quad \text { for every } x \in X .
$$

From this, it follows that

$$
v\left(x_{0}\right) p\left(\psi\left(x_{0}\right) y_{0}\right) \leq \frac{v\left(x_{0}\right) p\left(\psi\left(x_{0}\right) y_{0}\right)}{2}
$$

which is impossible and hence in this case our claim is established.

CASE (ii). Suppose $u\left(x_{0}\right) \neq 0, q\left(y_{0}\right)=0$ and $v\left(x_{0}\right) p\left(\psi\left(x_{0}\right) y_{0}\right)>0$. Put $\varepsilon=v\left(x_{0}\right) p\left(\psi\left(x_{0}\right) y_{0}\right) / 2$. Then $G=\left\{x \in X: u(x)<\varepsilon+u\left(x_{0}\right)\right\}$ is an open neighbourhood of $x_{0}$ and therefore by [6, Lemma 2], there exists $f \in C V_{b}(X)$ such that $0 \leq f \leq 1, f\left(x_{0}\right)=1$ and $f(X-G)=0$. Define $g(x)=f(x) y_{0}$, for every $x \in X$. Then clearly $g \in C V_{b}(X, T)$ and for every $p \in \operatorname{cs}(T), 0 \leq(p \circ g) \leq p\left(y_{0}\right),(p \circ g)\left(x_{0}\right)=p\left(y_{0}\right)$ and $(p \circ g)(X-G)=0$. Consider $h=\varepsilon^{-1} g$. Then $h \in B_{u, q}$ and this yields that $\psi h \in B_{v, p}$. This implies that $v(x) p(\psi(x) h(x)) \leq 1$, for every $x \in X$. From this, it follows that

$$
v(x) p(\psi(x) g(x)) \leq \frac{v\left(x_{0}\right) p\left(\psi\left(x_{0}\right) y_{0}\right)}{2}, \quad \text { for every } x \in X .
$$

Further, it implies that

$$
v\left(x_{0}\right) p\left(\psi\left(x_{0}\right) y_{0}\right) \leq \frac{v\left(x_{0}\right) p\left(\psi\left(x_{0}\right) y_{0}\right)}{2}
$$

which is impossible and hence in this case too our claim is established.

CASE (iii). Finally, suppose $u\left(x_{0}\right)=0$ and $q\left(y_{0}\right)=0$. Let $v\left(x_{0}\right) p\left(\psi\left(x_{0}\right) y_{0}\right)$ $>0$ and put $\varepsilon=v\left(x_{0}\right) p\left(\psi\left(x_{0}\right) y_{0}\right) / 2$. Then $G=\{x \in X: u(x)<\varepsilon\}$ is an open neighbourhood of $x_{0}$ and again by [6, Lemma 2], there exists $f \in$ $C V_{b}(X)$ such that $0 \leq f \leq 1, f\left(x_{0}\right)=1$ and $f(X-G)=0$. Define $g(x)=f(x) y_{0}$, for every $x \in X$. Then clearly $g \in C V_{b}(X, T)$ and for every 
$p \in \operatorname{cs}(T), 0 \leq(p \circ g) \leq p\left(y_{0}\right),(p \circ g)\left(x_{0}\right)=p\left(y_{0}\right)$ and $(p \circ g)(X-G)=0$. Consider $h=\varepsilon^{-1} g$. Then $h \in B_{u, q}$ and this implies that $\psi h \in B_{v, p}$. Hence $v(x) p(\psi(x) h(x)) \leq 1$, for every $x \in X$. From this, it follows that

$$
v(x) p(\psi(x) g(x)) \leq \frac{v\left(x_{0}\right) p\left(\psi\left(x_{0}\right) y_{0}\right)}{2}, \quad \text { for every } x \in X .
$$

Further, it implies that

$$
v\left(x_{0}\right) p\left(\psi\left(x_{0}\right) y_{0}\right) \leq \frac{v\left(x_{0}\right) p\left(\psi\left(x_{0}\right) y_{0}\right)}{2},
$$

which is a contradiction and with this our claim is established. This completes the proof of the theorem.

2.2 Remark (i). Every constant map $\psi: X \rightarrow B(T)$ induces a multiplication operator on $C V_{b}(X, T)$. For, if we define $\psi: X \rightarrow B(T)$ as $\psi(x)=A$, for every $x \in X$ where $A$ is any continuous linear operator on $T$. Let $v \in V$, and $p \in \operatorname{cs}(T)$. Since $A$ is a continuous linear operator, there exist $m>0$ and $q \in \operatorname{cs}(T)$ such that

$$
p(A y) \leq m q(y), \quad \text { for every } y \in T .
$$

This implies that $p(\psi(x) y) \leq m q(y)$, for every $x \in X$ and $y \in T$. Further, it follows that

$$
\begin{aligned}
v(x) p(\psi(x) y) & \leq m v(x) q(y) \quad(\text { for every } x \in X \text { and } y \in T) \\
& \leq u(x) q(y) \quad((\text { for every } x \in X \text { and } y \in T) .
\end{aligned}
$$

Hence by Theorem 2.1, $M_{\psi}$ is a multiplication operator on $C V_{b}(X, T)$.

(ii) Let $X$ be a completely regular Hausdorff space and let $T=Y$ be any Banach space. Then every continuous bounded operator-valued mapping induces a multiplication operator on $C V_{b}(X, Y)$. For, let $\psi: X \rightarrow B(Y)$ be a bounded operator-valued mapping. Then there exists $m>0$ such that $\|\psi(x)\| \leq m$, for every $x \in X$, Let $v \in V, x \in X$ and $y \in Y$. Then

$$
\begin{aligned}
v(x)\|\psi(x) y\| & \leq v(x)\|\psi(x)\|\|y\| \leq m v(x)\|y\| \\
& \leq u(x)\|y\| \quad \text { (for every } x \in X \text { and } y \in Y \text { ). }
\end{aligned}
$$

Hence by Theorem 2.1, $M_{\psi}$ is a multiplication operator on $C V_{b}(X, Y)$.

If $T=Y$ is any Banach space and $V$ is the system of weights generated by the characteristic functions of all compact subsets, then it turns out that every continuous operator-valued mapping induces a multiplication operator on $C V_{b}(X, Y)$. This we shall establish in the following proposition.

2.3 Proposition. Let $X$ be a completely regular Hausdorff space and let

$$
V=\left\{\lambda \chi_{K}: \lambda \geq 0, K \subset X \text { and } K \text { is a compact set }\right\} \text {. }
$$


Then every continuous mapping $\psi: X \rightarrow B(Y)$, induces a multiplication operator $M_{\psi}$ on $C V_{b}(X, Y)$.

Proof. In order to show that $M_{\psi}$ is a continuous linear operator on $C V_{b}(X, Y)$, in the light of Theorem 2.1 it is enough to show that for every $v \in V$, there exists $u \in V$ such that

$$
v(x)\|\psi(x) y\| \leq u(x)\|y\|, \quad \text { for every } x \in X \text { and } y \in Y \text {. }
$$

If $v \in V$, then $v=\lambda \chi_{K}$, for some compact subset $K$ of $X$. Since $\psi: X \rightarrow B(Y)$ is continuous, $\psi(K)$ is a compact subset in $B(Y)$. Let $m=\operatorname{Sup}\{\|\psi(x)\|: x \in K\}$. Put $u(x)=\lambda m \chi_{K}(x)$. Then $u \in V$. Let $x \in K$ and $y \in Y$. Then

$$
\|\psi(x) y\| \leq\|\psi(x)\|\|y\| \leq m\|y\| .
$$

From this, it follows that

$$
\lambda \chi_{K}(x)\|\psi(x) y\| \leq \lambda \chi_{K}(x) m\|y\| .
$$

This implies that

$$
v(x)\|\psi(x) y\| \leq u(x)\|y\|, \quad \text { for every } x \in K \text { and } y \in Y .
$$

On the other hand, if $x \in X \backslash K$, then obviously

$$
v(x)\|\psi(x) y\| \leq u(x)\|y\| .
$$

Thus $v(x)\|\psi(x) y\| \leq u(x)\|y\|$, for every $x \in X, y \in Y$ and hence $M_{\psi}$ is a multiplication operator on $C V_{b}(X, Y)$. This completes the proof of the theorem.

2.4 REMarK (i). From the above proposition, we note that if $\psi: X \rightarrow$ $B(T)$ where $T$ is any Banach space, is an unbounded continuous operatorvalued mapping, even then $\psi$ gives rise to a multiplication operator $M_{\psi}$ on $C V_{b}(X, T)$, where $V$ is the system of weights generated by the characteristic functions of all compact subsets of $X$.

(ii) In the above proposition, if we replace the system of weights

$$
V=\left\{\lambda \chi_{K}: \lambda \geq 0, K \subset X \text { and } K \text { is a compact set }\right\}
$$

by $C_{c}^{+}(X)$, the set of all positive continuous functions having compact supports, even then the conclusion holds.

2.5 Corollary. Let $X$ have the discrete topology and

$$
V=\left\{\lambda \chi_{K}: \lambda \geq 0, K \subset X \text { and } K \text { is a compact set }\right\} \text {. }
$$


Then every map $\psi: X \rightarrow B(T)$, where $T$ is a Banach space, induces a multiplication operator $M_{\psi}$ on $C V_{b}(X, T)$.

Now, we shall give certain examples of operator-valued mappings which induce and do not induce multiplication operators on $C V_{b}(X, T)$.

2.6 Example. Let $X=\mathbb{N}$ with discrete topology and let $T=l^{2}$, the Hilbert space of all square summable sequences of complex numbers. If we define $\psi: \mathbb{N} \rightarrow B\left(l^{2}\right)$ by $\psi(n)=U^{n}$, where $U$ is the unilateral shift operator on $l^{2}$, then

$$
\|\psi(n)\|=\left\|U^{n}\right\| \leq\|U\|^{n} \leq 1, \quad \text { for every } n \in \mathbb{N} .
$$

This shows that $\psi$ is a bounded operator-valued mapping and hence by Remark 2.2 (ii), $M_{\psi}$ is a multiplication operator on $C V_{b}(X, T)$.

2.7 ExAmple. Let $X=\mathbb{N}$, with discrete topology and $T=\mathbb{R}^{2}$, the real Banach space. Define $\psi: \mathbb{N} \rightarrow B\left(\mathbb{R}^{2}\right)$ by $\psi(n)=P^{n}$, where $P$ is a projection operator on $\mathbb{R}^{2}$. Then $\|\psi(n)\|=\left\|P^{n}\right\| \leq\|P\|^{n} \leq 1$. This implies that $\psi$ is a bounded operator-valued mapping and hence by Remark 2.2(ii), $M_{\psi}$ is a multiplication operator on $C V_{b}(X, T)$.

2.8 EXAMPLE. Let $X=\mathbb{N}$ be the set of natural numbers with discrete topology and let $V=K^{+}(\mathbb{N})$, the system of all positive constant weights. Let $T=C_{b}(\mathbb{N})=l^{\infty}$ be the Banach space of all bounded sequences of complex numbers and $B\left(l^{\infty}\right)$, the Banach algebra of bounded operators on $l^{\infty}$. Define $\psi: \mathbb{N} \rightarrow B\left(l^{\infty}\right)$ as $\psi(n)=C_{\phi^{n}}$, where $C_{\phi}: l^{\infty} \rightarrow l^{\infty}$ is the composition operator induced by a map $\phi: \mathbb{N} \rightarrow \mathbb{N}$. Then it can be seen that for every $v \in V$, there exists $u \in V$ such that

$$
v(n)\|\psi(n) f\| \leq u(n)\|f\|, \quad \text { for every } n \in \mathbb{N} \text { and } f \in l^{\infty}
$$

and hence by Theorem 2.1, $M_{\psi}$ is a multiplication operator on $C V_{b}(X, T)$.

2.9 EXAMPLE. Let $X=\mathbb{N}$, the set of natural numbers with discrete topology, $T=l^{2}$ and let $B\left(l^{2}\right)$ be the Banach space of bounded linear operators on $l^{2}$. Let $v(n)=n$, for every $n \in \mathbb{N}$. Then $V=\{\lambda v: \lambda \geq 0\}$ is a system of weights on $\mathbb{N}$. Let us define $\psi: \mathbb{N} \rightarrow B\left(l^{2}\right)$ as $\psi(n)=A^{n}$, where $A$ is the multiplication operator on $l^{2}$ induced by the constant function 2 , that is, $A: l^{2} \rightarrow l^{2}$ is defined as

$$
A\left(x_{1}, x_{2}, \ldots\right)=2\left(x_{1}, x_{2} \ldots\right) \text {. }
$$

Then clearly one can check that

$$
v(n)\|\psi(n) x\| \not \leq u(n)\|x\| .
$$

Thus $\psi$ does not induce a multiplication operator $M_{\psi}$ on $C V_{b}\left(\mathbb{N}, l^{2}\right)$. In fact $M_{\psi}$ is not even an into map. For, take $f: \mathbb{N} \rightarrow l^{2}$ as $f(n)=1 / n^{2}$. Then 
obviously $f \in C V_{b}\left(\mathbb{N}, l^{2}\right)$ but $\psi f(n)=\psi(n) f(n)=A^{n}\left(1 / n^{2}\right)=2^{n} / n^{2} \rightarrow \infty$ as $n \rightarrow \infty$ and therefore $\psi f \notin C V_{b}\left(\mathbb{N}, l^{2}\right)$. In this example, if we take $V$ as the system of positive constant weights on $\mathbb{N}$, even then $\psi$ does not induce a multiplication operator $M_{\psi}$ on $C V_{b}\left(\mathbb{N}, l^{2}\right)$. If fact, if $f(n)=1 / n$, then $f \in C V_{b}\left(\mathbb{N}, l^{2}\right)$ but $\psi f \notin C V_{b}\left(\mathbb{N}, l^{2}\right)$.

\section{Dynamical systems induced by multiplication operators}

Throughout this section we shall take $X$ to be the real line $\mathbb{R}$ (with the usual topology) and $T$ to be a Banach space. We shall denote by $B(T)$, the Banach algebra of all bounded linear operators on $T$ and by $F_{b}(\mathbb{R})$, the set of all continuous bounded functions on $\mathbb{R}$. Let $V$ be a system of weights on $\mathbb{R}$. Then clearly $C V_{b}(\mathbb{R}, T)$ is a locally convex Hausdorff topological vector space with the weighted topology defined in the last section. Now let $U$ be a countable set of non-negative upper semicontinuous functions on $\mathbb{R}$ such that $W=\{\lambda u: \lambda \geq 0, u \in U\}$ is a system of weights on $\mathbb{R}$ with $W \approx V$. Then one can easily prove that the weighted space $C V_{b}(\mathbb{R}, T)$ is metrizable. In case $T=\mathbb{C}$, the metrizable weighted space $C V_{b}(\mathbb{R})$ is a special case of the result proved by Summers [10, Theorem 2.10].

Now, fix $g \in F_{b}(\mathbb{R})$ and $A \in B(T)$. For each $t \in \mathbb{R}$, we define $\psi_{t}: \mathbb{R} \rightarrow$ $B(T)$ as $\psi_{t}(w)=e^{t g(w) A}$, for every $w \in \mathbb{R}$. We can easily see that $\psi_{t}$ is a bounded operator-valued mapping from $\mathbb{R} \rightarrow B(T)$ and hence by Remark 2.2(ii), $\psi_{t}$ induces a multiplication operator $M_{\psi_{t}}$ on the weighted metrizable locally convex Hausdorff space $C V_{b}(\mathbb{R}, T)$.

3.1 TheOREM. Let $g \in F_{b}(\mathbb{R}), A \in B(T)$ and let $\Pi_{A, g}: \mathbb{R} \times C V_{b}(\mathbb{R}, T)$ $\rightarrow C(\mathbb{R}, T)$ be the function defined by $\Pi_{A, g}(t, f)=M_{\psi_{t}} f$ for $t \in \mathbb{R}$ and $f \in C V_{b}(\mathbb{R}, T)$. Then $\Pi_{A, g}$ is a dynamical system on $C V_{b}(\mathbb{R}, T)$.

Proof. Since $M_{\psi_{t}}$ is a multiplication operator on $C V_{b}(\mathbb{R}, T)$ for every $t \in \mathbb{R}$, we can conclude that $\Pi_{A, g}(t, f)$ belongs to $C V_{b}(\mathbb{R}, T)$ whenever $t \in \mathbb{R}$ and $f \in C V_{b}(\mathbb{R}, T)$. Thus $\Pi_{A, g}$ is a function from $\mathbb{R} \times C V_{b}(\mathbb{R}, T)$ to $C V_{b}(\mathbb{R}, T)$. It can be easily seen that $\Pi_{A, g}(0, f)=f$, and

$$
\Pi_{A, g}(t+s, f)=\Pi_{A, g}\left(t, \Pi_{A, g}(s, f)\right)
$$

for all $t, s \in \mathbb{R}$ and $f \in C V_{b}(\mathbb{R}, T)$.

In order to show that $\Pi_{A, g}$ is a dynamical system on $C V_{b}(\mathbb{R}, T)$, it is enough to show that $\Pi_{A, g}$ is a separately continuous map since joint continuity follows from [3, Theorem 1]. Let us first prove the continuity of 
$\Pi_{A, g}$ in the first argument. Let $t_{n} \rightarrow t$ in $\mathbb{R}$. Then $\left|t_{n}-t\right| \rightarrow 0$ as $n \rightarrow \infty$. We shall show that $\Pi_{A, g}\left(t_{n}, f\right) \rightarrow \Pi_{A, g}(t, f)$ in $C V_{b}(\mathbb{R}, T)$. Let $v \in V$. Then

$$
\begin{aligned}
& \left\|\Pi_{A, g}\left(t_{n}, f\right)-\Pi_{A, g}(t, f)\right\|_{v}\|=\| \psi_{t_{n}} f-\psi_{t} f \|_{v} \\
& \quad=\operatorname{Sup}\left\{v(w)\left\|\left(\psi_{t_{n}}(w)-\psi_{t}(w)\right)(f(w))\right\|: w \in \mathbb{R}\right\} \\
& \quad=\operatorname{Sup}\left\{v(w)\left\|\left(e^{t_{n} g(w) A-t g(w) A}-I\right) e^{t g(w) A}(f(w))\right\|: w \in \mathbb{R}\right\} \\
& \quad \leq \operatorname{Sup}\left\{v(w)\left\|\left(e^{t_{n} g(w) A-t g(w) A}-I\right)\right\|\left\|e^{t g(w) A}(f(w))\right\|: w \in \mathbb{R}\right\} \\
& \quad \leq \operatorname{Sup}\left\{\left\|\left(e^{t_{n} g(w) A-t g(w) A}-I\right)\right\|: w \in \mathbb{R}\right\} \operatorname{Sup}\left\{v(w)\left\|e^{t g(w) A}(f(w))\right\|: w \in \mathbb{R}\right\} \\
& \quad \leq\left(e^{\left|t_{n}-t\right| M\|A\|}-1\right)\|f\|_{u} \rightarrow 0 \text { as }\left|t_{n}-t\right| \rightarrow 0 .
\end{aligned}
$$

This proves the continuity of $\Pi_{A, g}$ in the first argument. Now, we shall prove the continuity of $\Pi_{A, g}$ in the second argument. Let $\left\{f_{\alpha}\right\}$ be a net in $C V_{b}(\mathbb{R}, T)$ such that $f_{\alpha} \rightarrow f$ in $C V_{b}(\mathbb{R}, T)$. Then $\left\|f_{\alpha}-f\right\|_{v} \rightarrow 0$ for every $v \in V$. We shall show that

$$
\Pi_{A, g}\left(t, f_{\alpha}\right) \rightarrow \Pi_{A, g}(t, f) \text { in } C V_{b}(\mathbb{R}, T) .
$$

For this, let $v \in V$. Then

$$
\begin{aligned}
\left\|\Pi_{A, g}\left(t, f_{\alpha}\right)-\Pi_{A, g}(t, f)\right\|_{v} & =\left\|\psi_{t} f_{\alpha}-\psi_{t} f\right\|_{v} \\
& =\operatorname{Sup}\left\{v(w)\left\|\psi_{t}(w)\left(f_{\alpha}(w)-f(w)\right)\right\|: w \in \mathbb{R}\right\} \\
& \leq \operatorname{Sup}\left\{u(w)\left\|f_{\alpha}(w)-f(w)\right\|: w \in \mathbb{R}\right\} \\
& =\left\|f_{\alpha}-f\right\|_{u} \rightarrow 0 .
\end{aligned}
$$

This proves the continuity of $\Pi_{A, g}$ in the second argument and hence $\Pi_{A, g}$ is a (linear) dynamical system on the weighted space $C V_{b}(\mathbb{R}, T)$.

\section{References}

[1] K. D. Bierstedt, 'Gewichtete Raume Stetiger Vektorwertiger Funktionen und das Injektive Tensor-produkt I', J. reine u. angew Math. 259 (1973), 186-210.

[2] K. D. Bierstedt, 'Gewichtete Reume Stetiger Vektorwertiger Funktionen und das Injektive Tensor-produkt II', J. reine u. angew Math. 260 (1973), 133-146.

[3] P. Chernoff, and J. Marsden, 'On continuity and smoothness of group actions', Bull. Amer. Math. Soc. 76 (1970), 1044-1049.

[4] A. Gronthedieck, Topological Vector Spaces, (Gordon and Breach, New York, 1975).

[5] G. Kothe, Topological Vector Spaces I (Springer-Verlag, Berlin, 1969).

[6] L. Nachbin, Elements of Approximation Theory, Math. Studies 14, (Van Nostrand, Princeton, 1967). 
[7] J. B. Prolla, 'Weighted spaces of vector-valued continuous functions', Ann. Mat. Pura. Appl. 89 (1971), 145-158.

[8] R. K. Singh and Jasbir Singh Manhas, 'Multiplication operators on spaces of vectorvalued continuous functions', J. Austral. Math. Soc. (Series A), 50 (1991), 98-107.

[9] R. K. Singh and W. H. Summers, 'Composition operators on weighted spaces of continuous functions', J. Austral. Math. Soc. (Series A) 45 (1988), 303-319.

[10] W. H. Summers, Weighted locally convex spaces of continuous functions, Ph.D. Dissertation, Louisiana State University, 1968.

\section{University of Jammu}

Jammu-180004

India 